\title{
RF MEMS CAPACITOR INTEGRATION
}

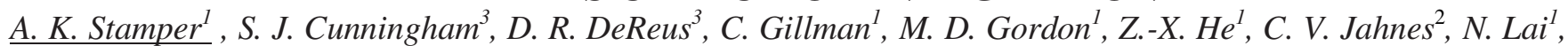 \\ S. L. Luce ${ }^{I}$, J. Maling ${ }^{1}$, A. S. Morris ${ }^{3}$, W. J. Murphy ${ }^{I}$, I. Vitomirov ${ }^{3}$, E. J. White ${ }^{I}$, and B. K. Wong ${ }^{I}$ \\ ${ }^{1}$ IBM, Inc., Essex Junction, VT USA, ${ }^{2}$ IBM, Inc. Yorktown Heights, NY USA \\ ${ }^{3}$ Wispry Inc., Irvine, CA USA
}

\begin{abstract}
MEMS capacitor switches have been integrated with $50 \mathrm{~V}$ LDMOS/5V-CMOS $0.18 \mu \mathrm{m}$ generation ICs. The MEMS were formed with the final three Ti-cladded $\mathrm{AlCu}$ wiring levels in $\mathrm{SiO}_{2}$ using a novel planar sacrificial silicon cavity process [1-3]. The MEMS cavities were hermetically sealed at less than atmospheric pressure at wafer level with a CVD dielectric lid. After Pb-free solder bumping, the MEMS chips are packaged in organic laminate packages. The capacitor portion of the MEMS beam has a capacitance density of $\sim 0.12 \mathrm{fF} / \mu^{2}$ and the pull-in, restoring, selfactuation, and break down voltages are on the order of $30 \mathrm{~V}, 10 \mathrm{~V}$, $>45 \mathrm{~V}$, and $>150 \mathrm{~V}$ for $0.2 \mathrm{pF}$ devices, respectively. MEMS cycling lifetime of 500 million cycles has been demonstrated. This paper summarizes the critical integration, yield, and reliability issues associated with the L1 qualification of this HV CMOS [4], RF MEMS technology.
\end{abstract}

\section{INTRODUCTION}

This is the first report of the L1 manufacturing qualification and early production ramp of a monolithically integrated RF CMOS-MEMS capacitor technology. The technology is in manufacturing production to provide chips for antenna tuning in cell phones. The MEMS capacitors are integrated in large preprogrammed tunable arrays with CMOS controllers and on-chip $40 \mathrm{~V}$ operation. Figure 1 shows an image of a 64 MEMS tunable capacitor chip. This paper summarizes the critical RF MEMS technology integration, yield, and reliability issues.

\section{MEMS TECHNOLOGY OVERVIEW \\ MEMS Capacitor Design and Fabrication}

The metal/oxide/metal MEMS beam is formed in a planar silicon cavity and the beam is released by removing the sacrificial silicon through holes in the oxide lid using $\mathrm{XeF}_{2}$ gas. The cavity is hermetically sealed by depositing CVD oxide and nitride to form a dielectric lid. The $\sim 70 \mu \mathrm{m}$ by $400 \mu \mathrm{m}$ asymmetric bridge MEMS beams are formed with two beams per cavity with nominal capacitances of $0.1,0.2$ or $0.4 \mathrm{pF}$ per beam. The MEMS capacitor electrodes are formed between the lower $\mathrm{AlCu}$ wire in the beam and the $\mathrm{AlCu}$ wire under the beam, both of which are coated with a thin MEMS capacitor dielectric to prevent aluminum-silicon reaction during fabrication and capacitor electrode shorting during actuation (Figures 2-3). 50nm tall oxide bumpers are formed inside patterned openings in the lower MEMS beam electrode (Figures 2-4) to prevent actuator-actuator contact, resulting in cycling lifetimes of $>500$ million cycles. The patterned openings in the lower beam electrode also act to reduce AlCu hillock density and height. The asymmetric actuators (Act) and RF capacitor (Cap) combined with the use of a longitudinal spring (Figure 5) act to minimize capacitance variability.

\section{MEMS Capacitor Electrode Topography}

We optimized the Ti-cladded AlCu metallurgy to reduce hillock density and height such that the average AFM-measured, random RMS roughness under the lower MEMS beam electrode and over the fixed electrode under the MEMS cavity are $2.3 \mathrm{~nm}$ and $4.3 \mathrm{~nm}$, respectively (Figure 6). We sporadically observe metal hillocks under the lower MEMS beam electrode, due to random process variability (Figures 7-8), and these hillocks can reduce capacitance density and act as cycling stiction nucleation points.

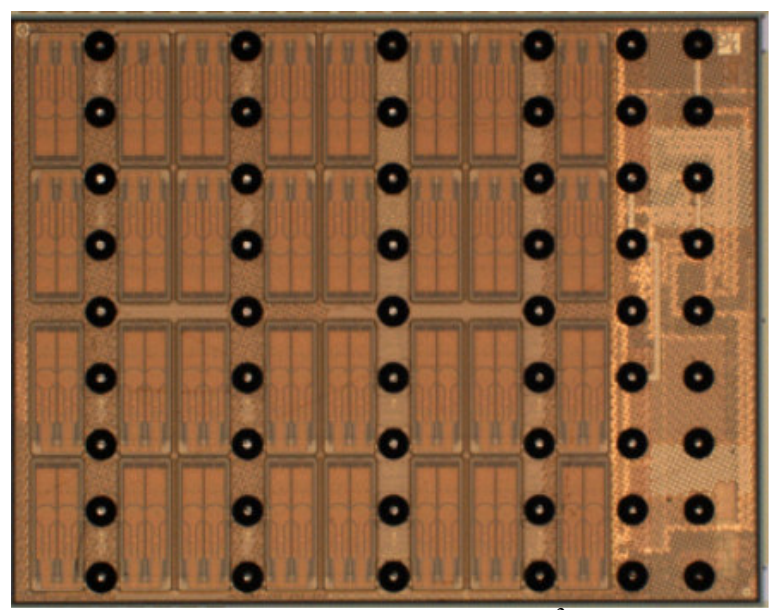

Figure 1: Optical image of $\sim 2.2 \times 2.8 \mathrm{~mm}^{2} 64$ MEMS capacitor Wispry tunable impedance matching chip post solder bumping.

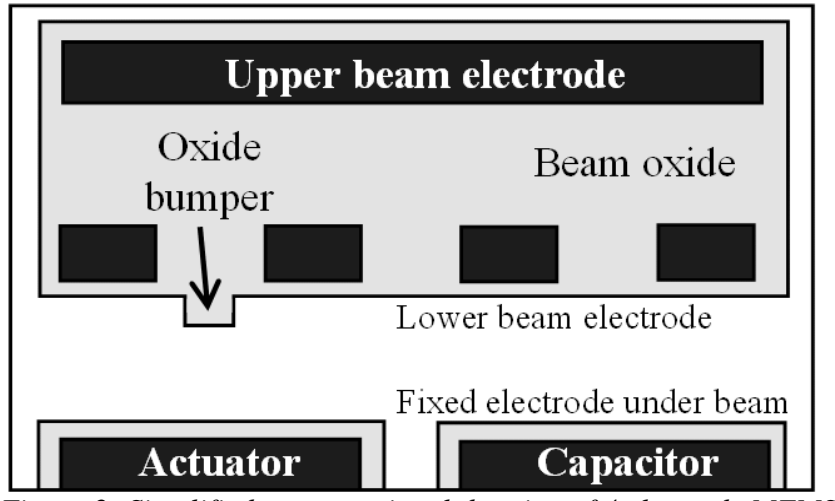

Figure 2: Simplified cross-sectional drawing of 4 electrode MEMS capacitor beam showing actuator and capacitor electrodes.

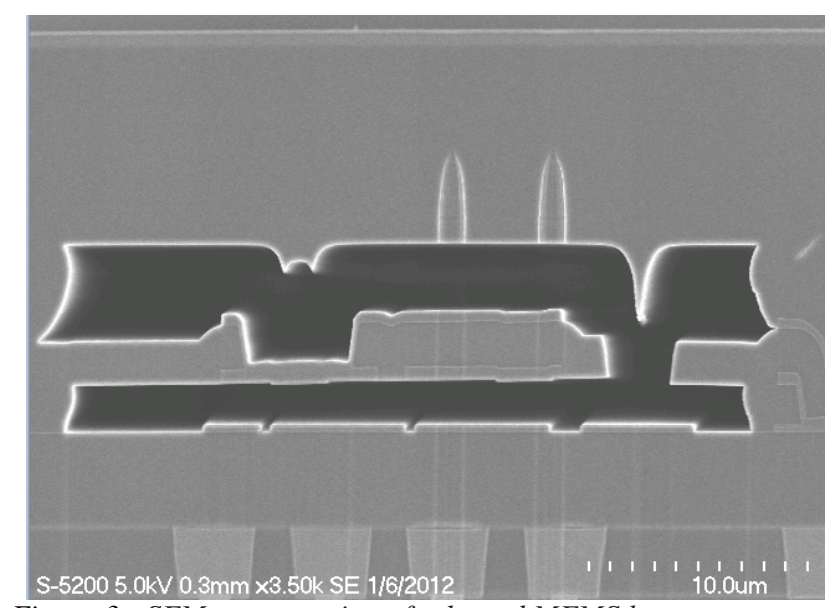

Figure 3: SEM cross-section of released MEMS beam test structure showing cavity, beam, and 10 1 m thick lid. 


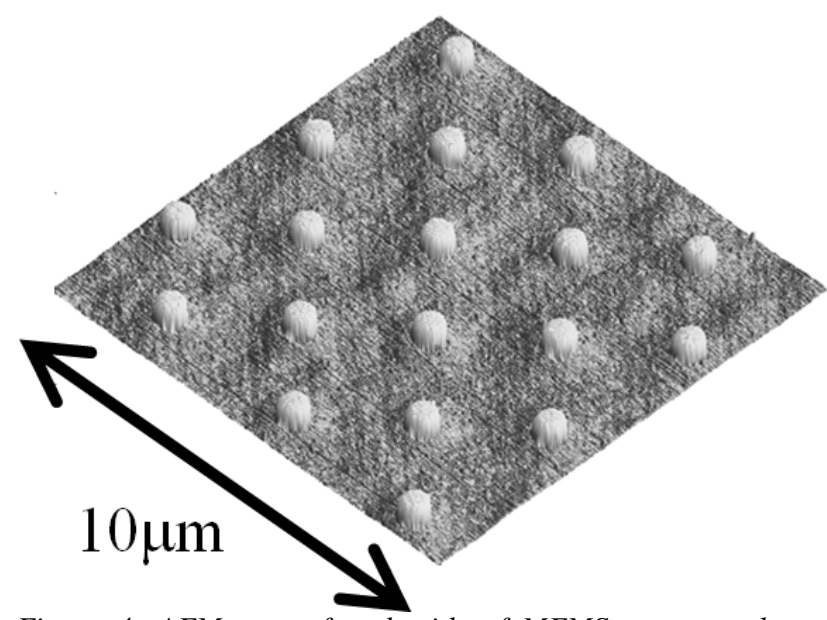

Figure 4: AFM map of underside of MEMS actuator electrode showing 50nm deep oxide bumper array (70nm min-max height).

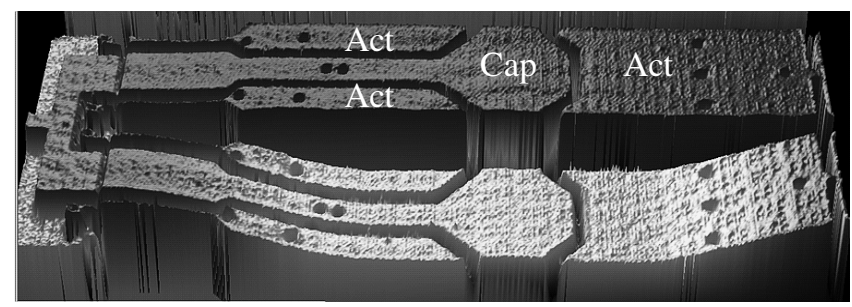

Figure 5: Optical profiler image of non-actuated (top) and actuated (bottom) MEMS capacitor beam including labels for the RF capacitor head (Cap) and actuators (Act).
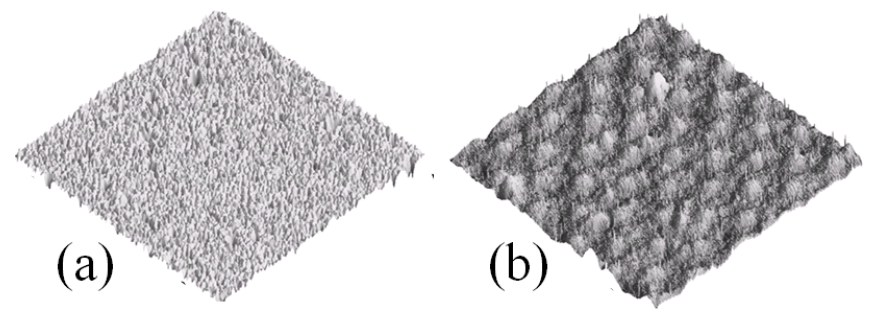

Figure 6: Typical AFM images for $400 \mu m^{2}$ areas (a) above the fixed electrode surface under the MEMS beam (63nm min-max) and (b) under the lower MEMS electrode (27nm min-max).

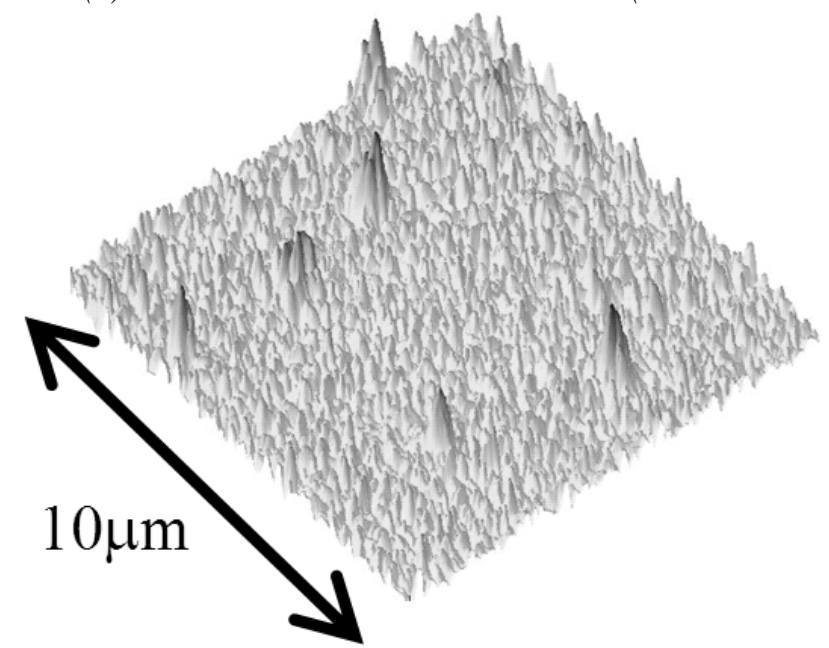

Figure 7: AFM roughness map of lower fixed MEMS capacitor electrode showing severe hillocks (64nm min-max height).

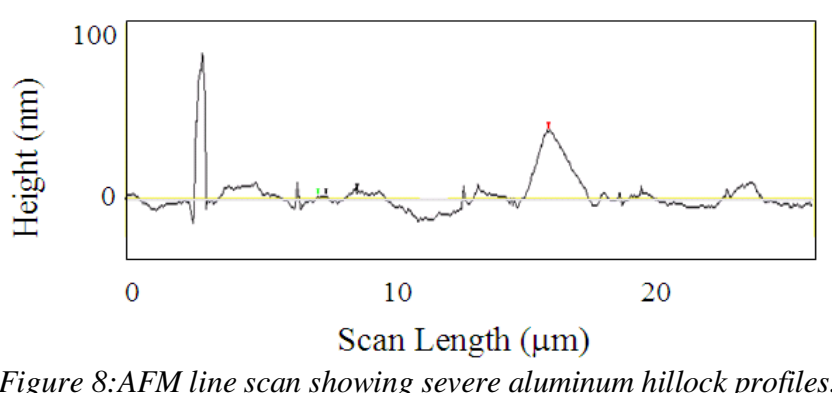

\section{MEMS CAPACITOR ELECTRICAL TEST}

The MEMS capacitor is operated by applying $40 \mathrm{~V}$ between the moving actuator electrodes and the fixed actuator electrodes under the MEMS beam (Figures 2 and 5). The operating voltage of $40 \mathrm{~V}$ is higher than the pull-in voltage. The MEMS beam consists of a metal/oxide/metal sandwich with oxide composing $~ 2 / 3$ of the volume. The low CTE of oxide combined with both the longitudinal spring at the end of the large actuators in the MEMS beam and the volume matching of the high CTE upper and lower MEMS beam metal act to minimize process- and temperatureinduced variability in beam shape. We measure $<2 \%$ capacitance and $<2 \mathrm{~V}$ pull-in voltage $-25^{\circ} \mathrm{C}$ to $100^{\circ} \mathrm{C}$ variability.

MEMS capacitance is defined as the $0-40 \mathrm{~V}$ delta capacitance difference (Figure 9). MEMS capacitor functional yields are determined by the delta capacitance and pull-in voltage (VPI) Figure 10 shows measured MEMS delta-capacitance and VPI data for $\sim 1 / 2$ year of early manufacturing production. The MEMS deltacapacitance has significantly increased in the past $1 / 2$ year, due to reduction of MEMS cavity residuals and metal hillocks on the MEMS capacitor electrodes. The primary functional yield loss mechanisms are delta-capacitance and VPI variability. VPI is proportional to (beam thickness) ${ }^{1.5}$ and (actuation gap) ${ }^{1.5}$ [5]. In addition, stress gradient changes in the MEMS beam induce positive or negative beam curvature which changes the actuation gap and resultant VPI. For example, a tensile beam stress gradient, i.e. having a more tensile film on the top of the beam, causes the beam center to bend downwards in a concave up fashion, resulting in reduced VPI due to a smaller actuation gap. Table 1 summarizes measured MEMS beam thickness and stress gradient variability and their effect on VPI variability for a MEMS capacitor centered with VPI $=30 \mathrm{~V}$. We actively modulate the expected released MEMS beam shape to center it at target by changing the thickness of the nominally $80 \mathrm{~nm}$ oxide thickness over the beam, which changes the MEMS beam stress gradient and resultant beam curvature. Figure 11 shows a 5V VPI shift per $10 \mathrm{~nm}$ of oxide thickness over the beam, primarily because of increased beam curvature and resultant air gap change.

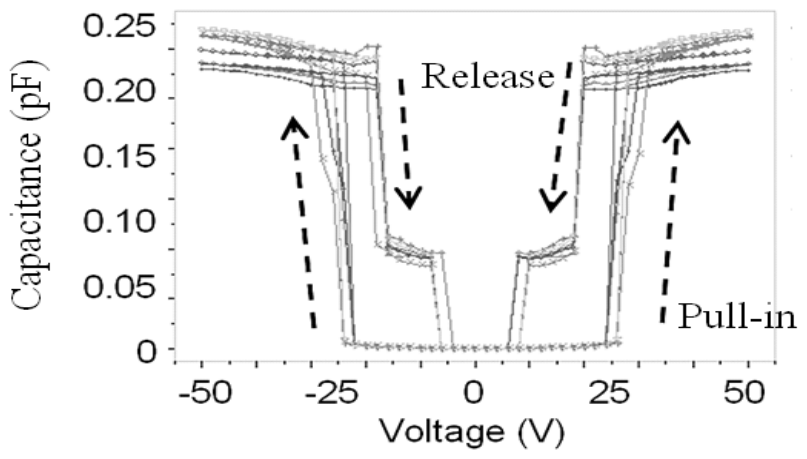

Figure 9: $M E M S$ bipolar $C$-V data for seven individual $0.2 p F$ wafer center, middle, and edge radius capacitors. 
In addition to the obvious problem of no MEMS capacitor actuation when VPI is greater than the $40 \mathrm{~V}$ operation voltage, a low VPI causes yield loss for self-actuation. Self-actuation occurs because the actuation force is proportional to (voltage) ${ }^{2}$ and the RF voltage across an unactuated MEMS capacitor causes it to actuate. For the MEMS design and test data shown in this paper, selfactuation falls below specification (40V) when VPI is below $\sim 26 \mathrm{~V}$.
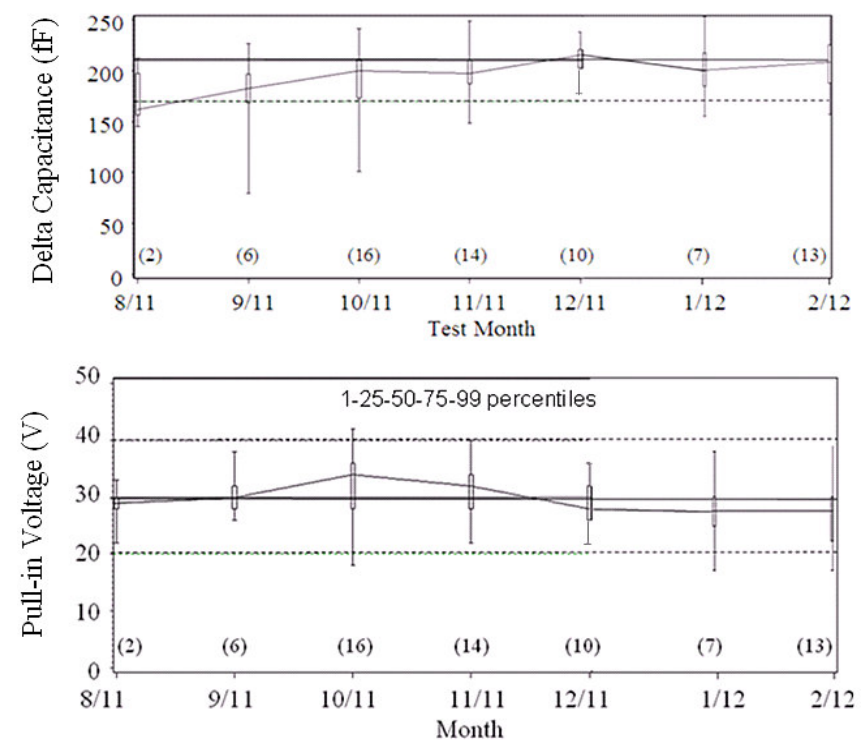

Figure 10: 200fF MEMS capacitor delta capacitance and pull-in voltage data for $\sim 70$ production lots with 6-25 wafers/lot and 10 chips/wafer (lots/month shown in parenthesis).

Table 1: MEMS beam process effect on pull-in voltage variability.

\begin{tabular}{|c|c|c|}
\hline Parameter & $\begin{array}{c}\text { Measured } \\
\text { Variability }\end{array}$ & $\begin{array}{c}\text { Predicted VPI } \\
\text { variability }\end{array}$ \\
\hline Beam oxide stress gradient & $28+/-6 \mathrm{MPa} / \mu \mathrm{m}$ & $+/-2.7$ \\
Beam oxide thickness & $2+/-0.1 \mu \mathrm{m}$ & $+/-2.3$ \\
\hline Beam metal thickness & $+/-20 \mathrm{~nm}$ & $+/-3.0$ \\
\hline Under/over beam ox thick & $80+/-5 \mathrm{~nm}$ & $+/-2.5$ \\
Under/over beam ox stress & $280+/-14 \mathrm{MPa}$ & $+/-1.0$ \\
\hline Lower cavity height & $1.9+/-0.2 \mu \mathrm{m}$ & $+/-4.4$ \\
\hline Total VPI variability & $+/-7 \mathrm{~V}$ & $+/-6.9$ \\
\hline
\end{tabular}

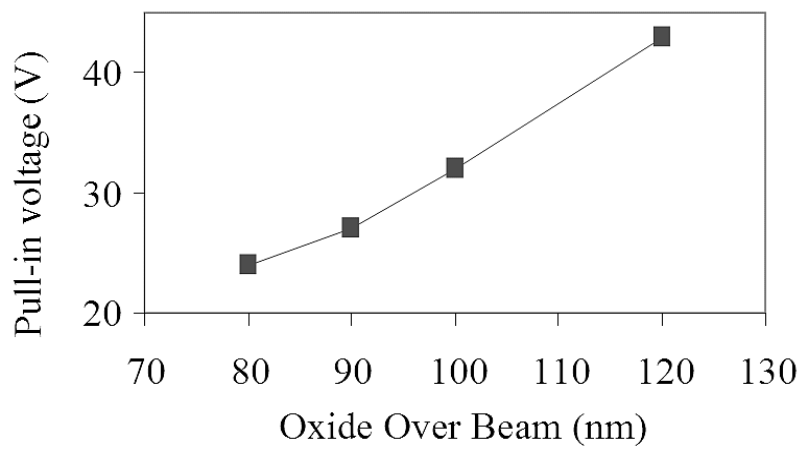

Figure 11: VPI vs. oxide thickness over the MEMS beam.

\section{MEMS Cavity Integrity}

Upon exiting the fab, wafers are processed through standard $\mathrm{Pb}$-free bump flip-chip assembly processes that include passivation, bumping, test, back-side grind, back-side coating, and dicing. The chips are then flip-chip mounted to an organic laminate carrier substrate with under fill and finally the substrate is mounted to a printed circuit board. Figure 12 shows MEMS cavity cracks that occurred on $\sim 6 \mathrm{~mm}^{2}$ packaged chips with 32 MEMS cavities per chip. We determined that these lid cracks became visible during back side grind and chip dicing and were exacerbated by the acute angle in the corner of the upper MEMS cavity (Figure 12). Lid cracks in packaged chips destroy the hermetic seal, which results in immediate stiction of the MEMS beam during electrical test. Lid cracking, which was modeled using finite element analysis, was eliminated by increasing the lid thickness from $6 \mu \mathrm{m}$ to $10 \mu \mathrm{m}$, which reduced the modeled maximum principle stress by a factor of $\sim 2.8$ and also reduced the $130^{\circ} \mathrm{C}, 91 \%$ R.H. pressure cooker stress fail rate of packaged chips from $\sim 20 \%$ to $0 \%$.

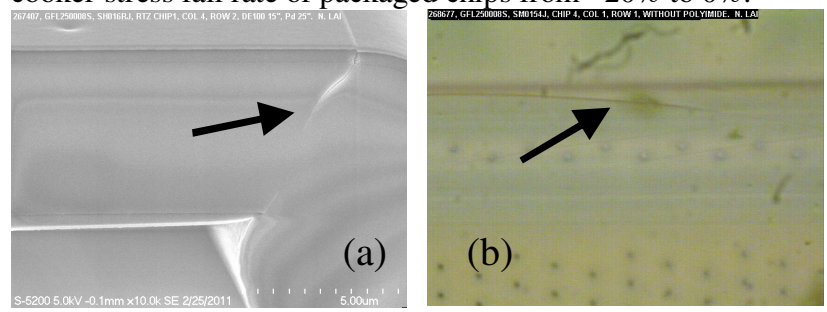

Figure 12: (a) SEM cross-section and (b) optical top view micrograph showing depackaged MEMS chip cavity cracks originating in cavity corner with $6 \mu$ m thick lid.

\section{MEMS CAPACITOR RELIABILTY Cycling}

MEMS capacitor cycling is a challenging reliability stress. End user specifications are variable but typically are 0.1 to 1 billion cycles over the operating temperature. Historically, we observed two primary cycling fail modes: 0V stiction-induced capacitance increase and $40 \mathrm{~V}$ defect- or wear-in-induced capacitance change [1]. Figure 13 shows representative cycling data for a single MEMS capacitor driver which connects 7 MEMS capacitors in parallel on a chip with 64 MEMS capacitors. Most drivers do not fail during our cycling tests (Chip 1) and, when they do, they fail for actuator stiction on one or more of the seven MEMS capacitors (Chip 2), which increases the $0 \mathrm{~V}$ capacitance. 2 of the 7 MEMS capacitors in Chip 2 exhibited actuator stiction fails, the first at 290 and a second at 350 million cycles. We sometimes observe a simultaneous $0 \mathrm{~V}$ and $40 \mathrm{~V}$ increase in capacitance (Chip 3), which may mean that an actuator stiction fail caused the actuated beam shape to change, resulting in a $40 \mathrm{~V}$ capacitance change. Cycling fails are rarely accompanied by dielectric breakdown, which means that the MEMS capacitor drivers continue to operate after the initial cycling fail. By cycling large numbers of chips and drivers, we calculated a fail rate of 1000 ppm at 500 million cycles.

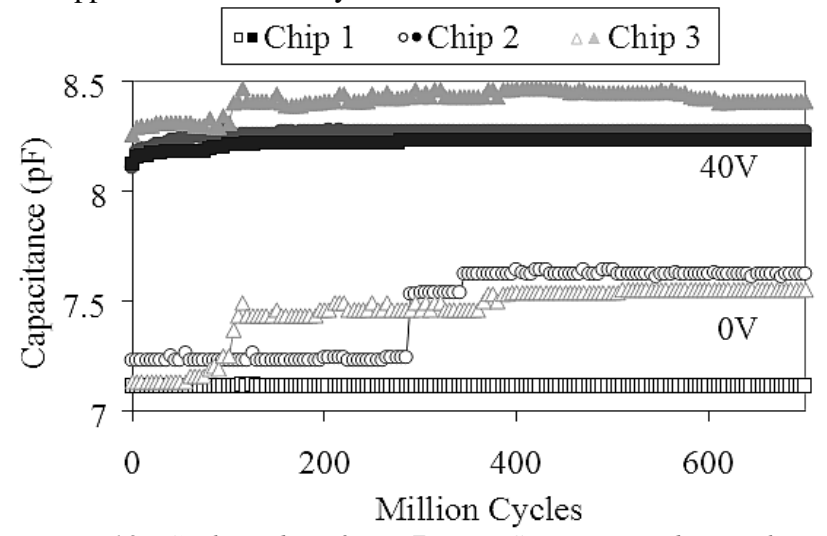

Figure 13: Cycling data for a 7 MEMS capacitor driver showing OV (open symbol) and 4OV (closed symbol) capacitance. 
Failure analysis of cycling fails (Figure 14) consistently shows actuator stiction in areas between the oxide bumpers. We believe that this stiction is due to the random presence of metal hillocks under the MEMS beam, which leads to electric field enhancement and accelerated stiction fails. These metal hillocks provide a high electric field actuator stiction point during cycling and the likelihood of their causing a stiction fail increases over time as the oxide bumpers are eroded. Figure 15 shows a severe case of hillocks under the MEMS beam.
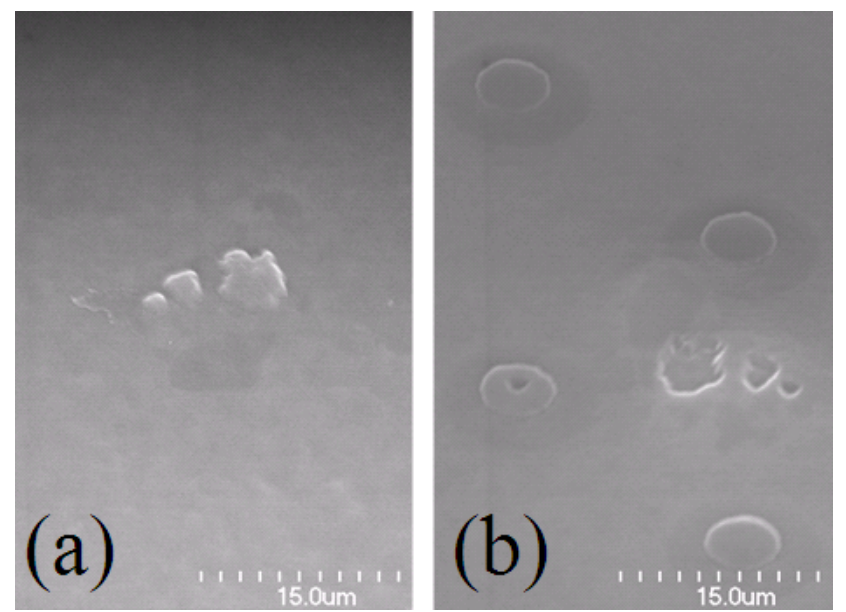

Figure 14: Typical MEMS cycling stiction fail showing (1) top side of fixed electrode and $(b)$ underside of movable electrode.

\section{MEMS Capacitor breakdown voltage}

The MEMS capacitor dielectric needs to be reliable up to $40 \mathrm{~V}$ DC actuation voltage and $80 \mathrm{~V}$ peak-to-peak RF voltage. Figure 16 shows measured actuated MEMS capacitor voltage breakdown (VBD) for the MEMS bridge beam used in the tunable capacitor chip (Figure 5). VBD is centered at about $150 \mathrm{~V}$ with little waferto- wafer and lot-to-lot variability and exceeds the $80 \mathrm{~V}$ specification. We measure both capacitor to capacitor and ganged actuator-capacitor to actuator-capacitor electrode VBD and observe slightly lower values for the ganged actuator-capacitor measurement, as expected from the larger area.

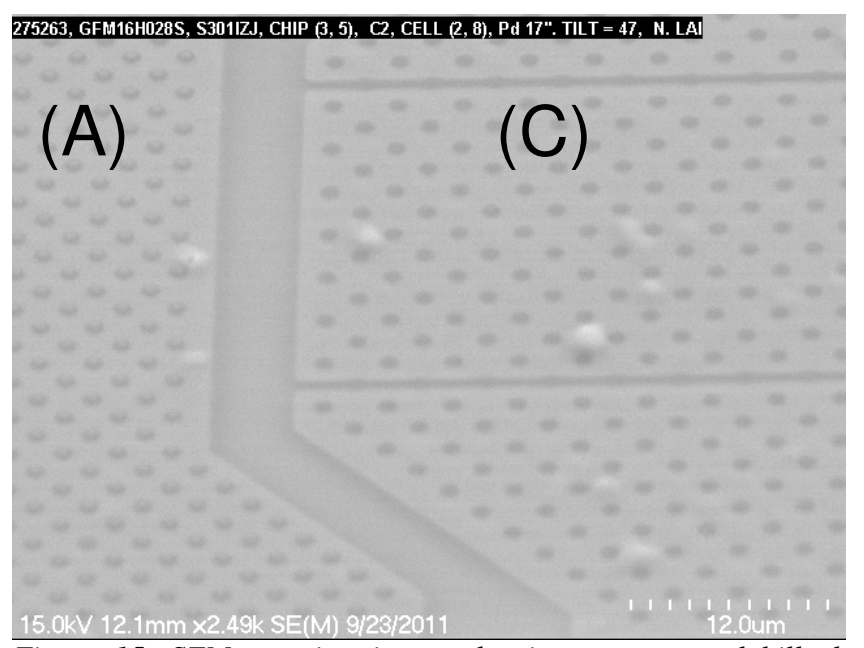

Figure 15: SEM top view image showing severe metal hillocks under the MEMS beam electrode on the actuator $(A)$ and capacitor (C) electrodes.

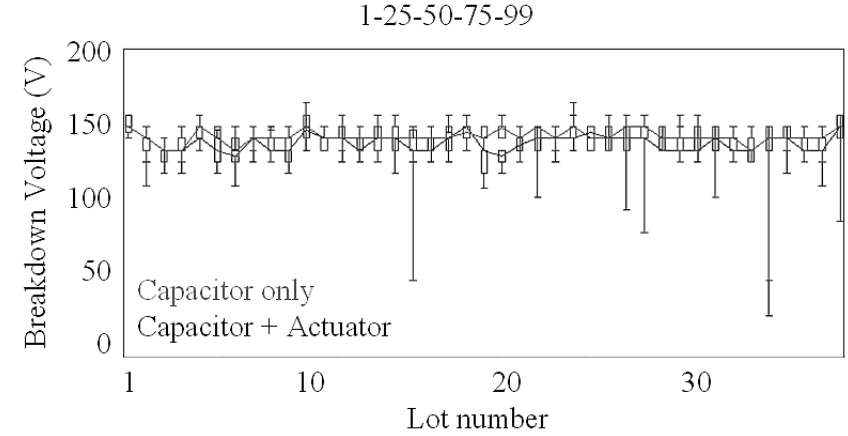

Figure 16: MEMS capacitor breakdown voltage.

\section{SUMMARY}

We have successfully L1 production qualified RF-MEMS capacitor switches into a $0.18 \mu \mathrm{m}$ high voltage CMOS process flow and begun the early manufacturing ramp. The MEMS capacitors were formed using a sacrificial silicon cavity process are hermetically sealed at wafer level, and flip chip packaged in organic laminate packages. No fails occurred during $130^{\circ} \mathrm{C}, 91 \%$ R.H. pressure cooker stressing of packaged chips. MEMS cycling lifetime of 500 million cycles has been demonstrated. The primary functional yield limiters are due to pull-in voltage and MEMS capacitance variability. This RF-MEMS technology has demonstrated preliminary yield and reliability sufficient for the initial application of cell phone antenna tuning.

\section{REFERENCES}

[1] A. K. Stamper, C. V. Jahnes, S. R. Dupuis, A. Gupta, Z.-X. He, R. T. Herrin, S. L. Luce, J. Maling, D. R. Miga, W. J. Murphy, E. J. White, S. Cunningham, D. R. DeReus, I. Vitomirov, and A. S. Morris, "Planar MEMS RF Capacitor Integration" in Proc. $16^{\text {th }}$ Transducers, Beijing, China, June 59, 2011, pp.1803-6.

[2] C. V. Jahnes, N. Hoivik, J. M. Cotte, M. Lu, and J. H. Magerlein, "Evaluation of O2 Plasma and XeF2 Vapor Etch Release Process for RF MEMS, Switches Fabricated using CMOS Interconnect Processes" in Proc. Solid State Sensors, Actuators, and Microsystems Workshop, Hilton Head Island, SC, United States, June 4-8, 2006 pp. 360-3.

[3] S. Natarajan, S. Cunningham, A. Morris, \& D. DeReus, "CMOS Integrated Digital RF MEMS Capacitors", $11^{\text {th }}$ Meeting on Silicon Monolithic Integrated Circuits in RF Systems, Phoenix, AZ, Jan. 17-19, 2011 pp. 173-176.

[4] R. Minixhofer, N. Feilchenfeld, M. Knaipp, G. Röhrer, J. M. Park, M. Zierak, H. Enichlmair, M. Levy, B. Löffler, D. Hershberger, F. Unterleitner, M. Gautsch, K. Chatty, Y. Shi, W. Posch, E. Seebacher, M. Schrems, J. Dunn, and D. Harame, "A 120V 180nm High Voltage CMOS smart power technology for System-on-chip integration", Proc. 22nd Int. Symp. Power Semicond. Dev. \& IC's, Hiroshima, Japan, June 7-10, 2010 pp. 75-78.

[5] Peter M. Osterberg and Stephen D. Senturia in J. Micromechanical Systems, Vol. 6, No. 2, June 1997.

\section{CONTACT}

*A. K. Stamper, tel: +1-802-769-8466; astamper@us.ibm.com 